\title{
AD-1775 - Antibiotics, paracetamol and infections during prenatal and early life in relation to type 1 diabetes
}

\author{
German Tapia $^{1^{*}}$, Ketil Størdal $1^{1,2^{*}}$, Karl Mårild ${ }^{1}$, Christian R. Kahrs², Torild \\ Skrivaraug $^{3}$, Pål R. Njølstad ${ }^{4,5}$, Geir Joner $^{3,6}$, Lars C. Stene ${ }^{1}$
}

\begin{abstract}
${ }^{1}$ Norwegian Institute of Public Health; ${ }^{2}$ Pediatric Department, Østfold Hospital Trust, Fredrikstad; 3 Pediatric Department, Oslo University Hospital, Oslo, Norway; ${ }^{4}$ KG Jebsen Center for Diabetes Research, Department of Clinical Science, University of Bergen, Bergen; 5 Department of Paediatrics, Haukeland University Hospital, Bergen; ${ }^{6}$ Institute of Clinical Medicine, University of Oslo, Oslo. *Shared first authorship
\end{abstract}

Aim: To investigate whether use of antibiotics or paracetamol, or infections in early childhood and in the mother during pregnancy are associated with increased risk of type 1 diabetes (T1D) in children.

\section{Subjects and Methods:}

Participants in the Norwegian Mother and Child (MoBa) cohort study reported infections and medication use through repeated questionnaires in pregnancy and until child's age 18 months. Antibiotic use was also studied in a larger cohort (MBRN) using nation wide registers (Figure 1).

Results: Antibiotic use in pregnancy, or child's use in early childhood, was not associated with risk of T1D (Figure 2).

Figure 2: Antibiotic use and offspring T1D

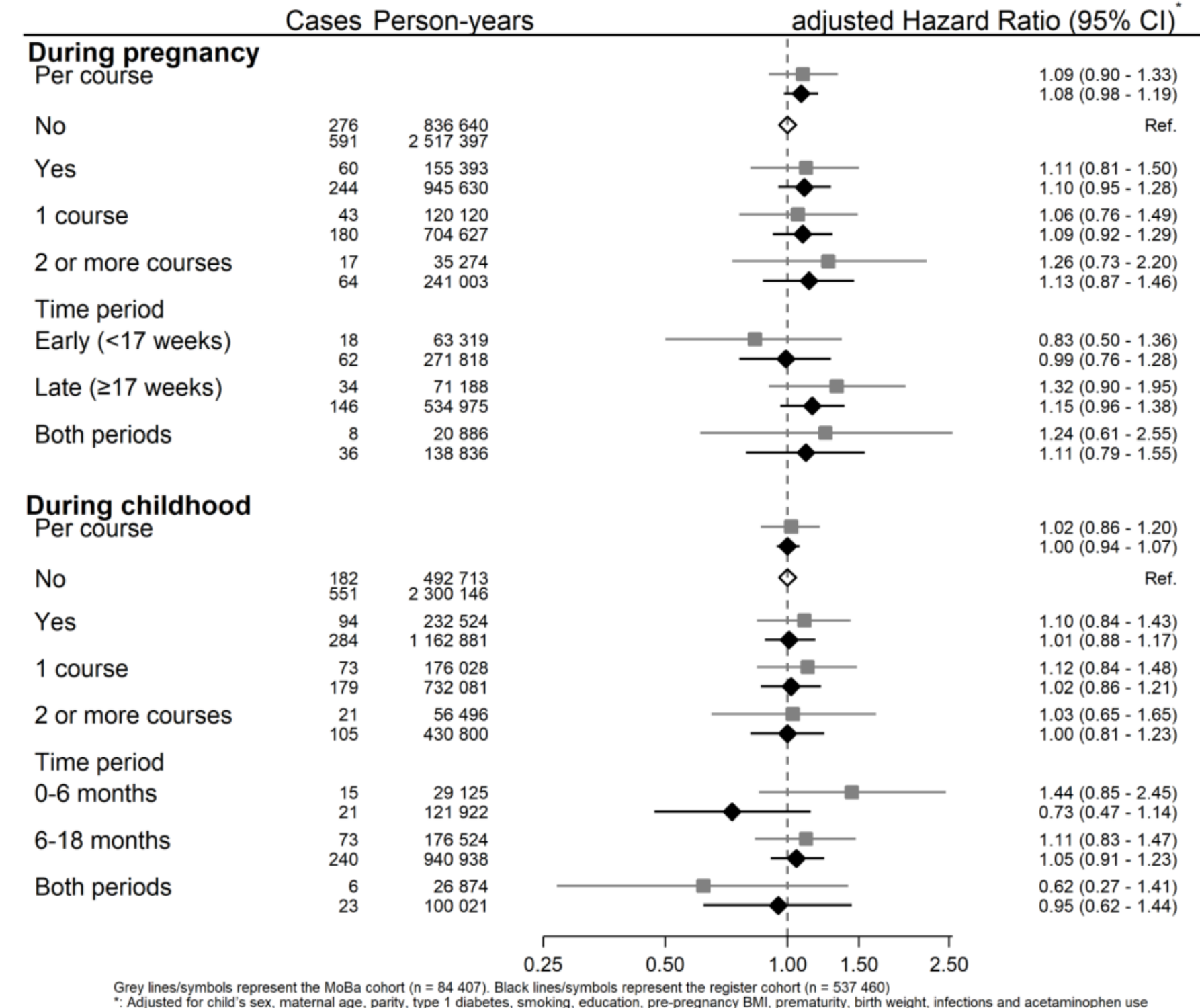

Hospitalization for gastroenteritis during the first 18 months of life was associated with increased risk (aHR 2.27, 95\% Cl 1.20-4.29) of T1D, while other infections or acetaminophen use during childhood was not associated with T1D (Figure 3).

Maternal infections or acetaminophen use during pregnancy did not predict offspring risk of T1D (data not shown).

\section{Conclusions:}

We did not find support for general early life infections, infection frequency nor use of antibiotics or acetaminophen to play a major role in childhood T1D.
Figure 1:

Flowchart of participants

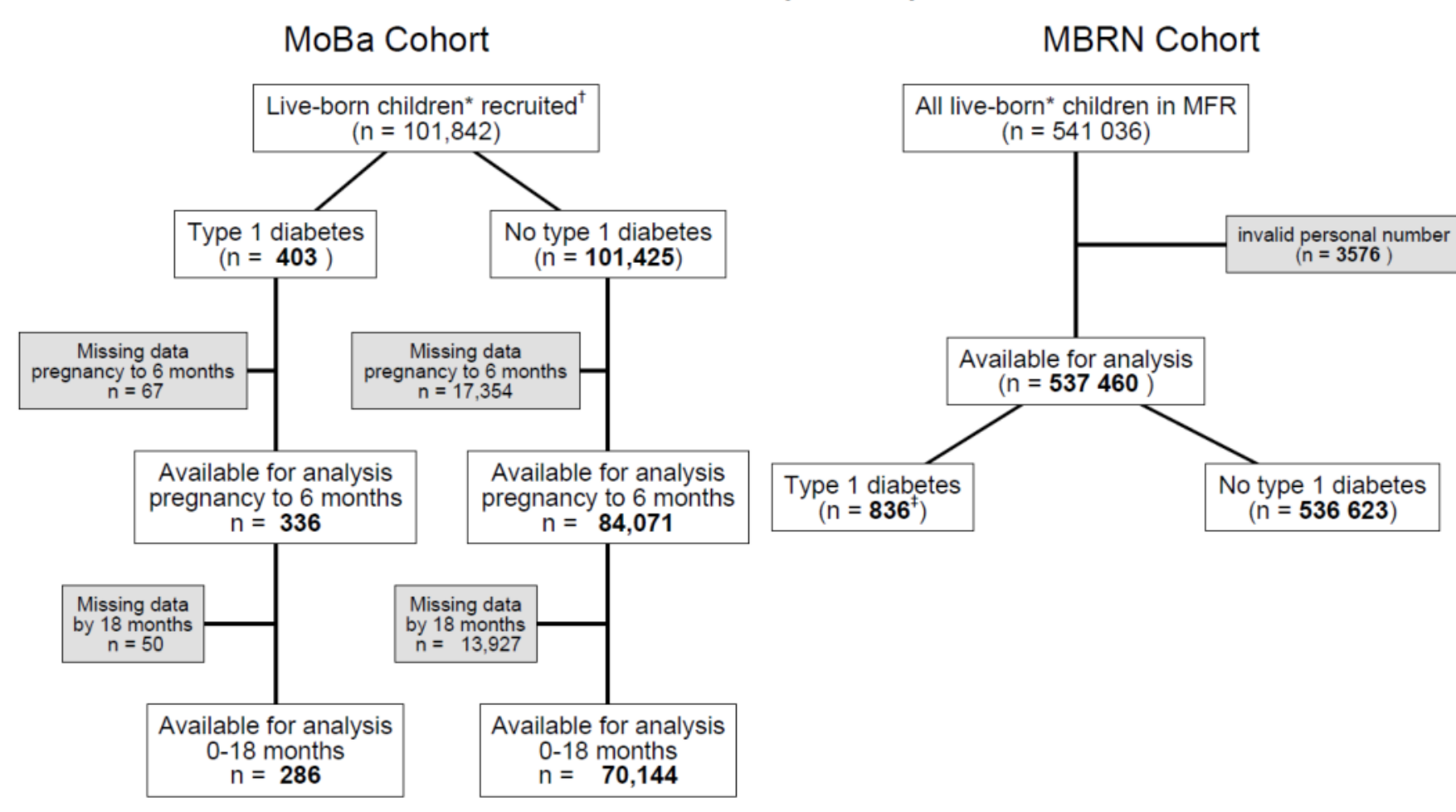

Figure 3: Infections and acetaminophen use and offspring T1D Exposure 0-18 months Cases Person-years adjusted Hazard Ratio $(95 \% \mathrm{Cl})$

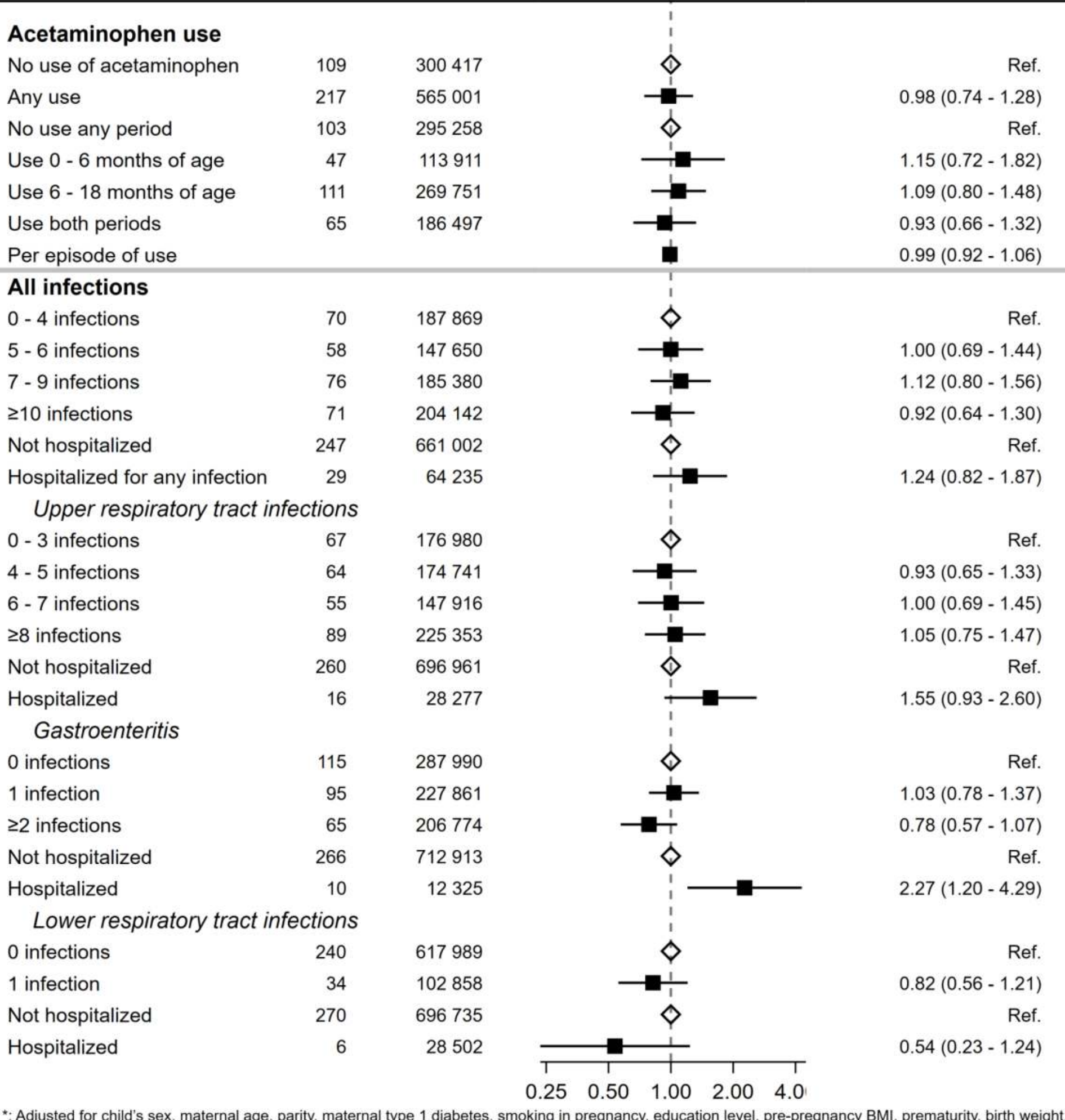

